Philosophie ANTIQUE

\section{Philosophie antique}

Problèmes, Renaissances, Usages

18 | 2018

L'athéisme antique

\title{
Aristote et la question de la complétude
}

Le modèle formel de Kurt Ebbinghaus

\section{Clément Lion et Shahid Rahman}

\section{(2) OpenEdition}

Journals

Édition électronique

URL : https://journals.openedition.org/philosant/1055

DOI : $10.4000 /$ philosant. 1055

ISSN : 2648-2789

Éditeur

Éditions Vrin

\section{Édition imprimée}

Date de publication : 1 novembre 2018

Pagination : 219-243

ISBN : 978-2-7574-2372-1

ISSN : 1634-4561

\section{Référence électronique}

Clément Lion et Shahid Rahman, «Aristote et la question de la complétude », Philosophie antique [En ligne], 18 | 2018, mis en ligne le 01 novembre 2019, consulté le 02 décembre 2022. URL : http:// journals.openedition.org/philosant/1055; DOI : https://doi.org/10.4000/philosant.1055

\section{(c) (i) (3)}

Creative Commons - Attribution - Pas d'Utilisation Commerciale - Pas de Modification 4.0 International - CC BY-NC-ND 4.0

https://creativecommons.org/licenses/by-nc-nd/4.0/ 


\author{
ARISTOTE ET LA QUESTION DE LA COMPLÉTUDE \\ Le modèle formel de Kurt Ebbinghaus \\ Clément Lion, Shahid RaHman \\ Univ. Lille, CNRS, UMR 8163 -STL- Savoirs Textes Langage
}

RÉSUMÉ. Avec l'article « Aristotle's natural deduction system », publié en 1974, J. Corcoran a contribué à diffuser une nouvelle perspective sur les écrits logiques d'Aristote et sur la théorie du syllogisme en particulier. Dans cet article, Corcoran affirme que, dans les premiers chapitres des Premiers Analytiques, Aristote ne propose pas un système axiomatique, qui supposerait une logique sous-jacente, ainsi que le pensait Łukasiewicz, mais plutôt un système de déduction naturelle, avec des dimensions métalogiques. Notre propos est ici basé sur une courte monographie de Kurt Ebbinghaus, intitulée Ein formales Model der Syllogistik des Aristoteles (1964), où est fixé le canon de cette nouvelle perspective mentionnée plus haut et qui a été développé dans le cadre conceptuel de la « logique opérative » de Paul Lorenzen. Ebbinghaus développe une reconstruction formelle montrant que l'approche d'Aristote relève de la « théorie de la preuve », non seulement pour ce qui concerne le système d'inférence sous-jacent, mais aussi pour ce qui concerne les éléments métalogiques. C'est notamment à travers ce dernier aspect que se manifeste une différence majeure par rapport à la reconstruction de Corcoran. Alors que celui-ci pose que le système d'inférence d'Aristote est enraciné dans une sémantique de théorie des modèles (élaborée par Corcoran lui-même), Ebbinghaus comprend que la théorie du syllogisme a été développée à partir d'une approche de la signification par des règles («rule based »), semblables aux règles d'un jeu. En fait, la reconstruction d'Ebbinghaus offre une lecture pragmatiste de la syllogistique d'Aristote, qui, tel est notre propos, paraît non seulement beaucoup plus proche du point de vue d'Aristote que ne l'est la sémantique de théorie des modèles proposée par Corcoran, mais qui, de plus, permet de saisir l'unité systématique de la théorie du syllogisme

Summary. With the article "Aristotle's natural deduction system", published in 1974, J. Corcoran has contributed to spread a new insight into Aristotelian logical writings and the theory of assertoric syllogism in particular. The main claim of this article is that, in the first chapters of the Prior Analytics, Aristotle doesn't expose an axiomatic system that would suppose an implicit underlying logic, as thought Eukasiewicz, but a natural deduction system, with metalogical dimensions. Ourpaper is based on a shortmonograph of Kurt Ebbinghaus, called Ein formales Model der Syllogistik des Aristoteles (1964),

Philosophie antique, ${ }^{\circ} 18$ (2018), 219-243 
which sets a landmark for the new insight mentioned above and that was developed within the conceptual framework of Paul Lorenzen's "operative logic". Ebbinghaus develops a formal reconstruction that shows that Aristotle's perspective is a proof-theoretical one, not only in relation to the underlying inference system but in relation to the study of its metalogical features. The latter indicates a main distinction to Corcoran's own reconstruction. Indeed, while Corcoran assumes that Aristotle's inferential systems is rooted on an underlying model-theoretical semantics (worked out by Corcoran himself), Ebbinghaus understands the theory of syllogism has been developed under the background of a "rule-based" approach to meaning similar to "game rules". In fact, Ebbinghaus' reconstruction proposes a pragmatist reading of Aristotle's syllogistic, that, so we claim, not only seems to be definitely closer to Aristotle's view, than the model-theoretical semantics developed by Corcoran, but also yields the theory of syllogism as a unified system. 
Dans la seconde moitié $\mathrm{du} \mathrm{Xx}^{\mathrm{e}}$ siècle, après avoir été complètement délaissée par les logiciens, la théorie aristotélicienne du syllogisme assertorique est de nouveau apparue comme un objet d'étude digne d'intérêt*. On considère généralement que ce retour paradoxal à la logique d'Aristote, si décriée à la fin du $\mathrm{XIX}^{\mathrm{e}}$ siècle, a procédé en deux temps principaux. Dans les années 50, J. Łukasiewicz proposa d'abord, dans son ouvrage Aristotle's Syllogistic from the Standpoint of Modern Formal Logic, de revenir au texte aristotélicien, par-delà la lecture traditionnelle; puis il montra que les résultats obtenus par Aristote pouvaient être retrouvés et complétés au moyen des outils de la logique formelle moderne (notamment le calcul des propositions et la théorie des quantificateurs), certes au prix d'un décalage important avec les procédés explicitement employés par Aristote lui-même, laissant ouvertes de nombreuses questions, dont G. Patzig dressa l'inventaire dans son propre ouvrage de 1959, Die aristotelische Syllogistik. Puis, au début des années 70, à travers une série d'articles ${ }^{1}$, J. Corcoran déploya une autre perspective, consistant à lire le début des Premiers Analytiques comme l'exposé d'un système de déduction naturelle autonome, même s'il devait être associé à une sémantique de théorie des modèles, demeurée implicite chez Aristote. La principale contribution de Corcoran fut de proposer une notion de la validité des syllo-

* Nous exprimons notre gratitude et nos plus vifs remerciements au professeur Michel Crubellier pour ses précieuses indications et pour la relecture minutieuse de la traduction de l'article d'Ebbinghaus par Clément Lion. Merci également à Zoé McConaughey pour les échanges fructueux dans le cadre d'un séminaire organisé au sein du département de philosophie de l'Université de Lille, portant sur les reconstructions modernes de la théorie du syllogisme d'Aristote et à l'occasion duquel s'est esquissé le contenu du présent article. Merci à la MESHS de la région Hauts-de-France, pour nous avoir donné l'occasion, dans le cadre de l'axe de recherche ADA (Argumenter-Décider-Agir), de soumettre une première présentation du contenu de cet article au jugement de chercheurs avisés. Merci à Thomas Bénatouil, pour nous avoir proposé de soumettre cet article à la revue Philosophie Antique. Merci enfin aux rapporteurs, dont les remarques nous ont permis de préciser certains points et de rendre ainsi notre propos, nous l'espérons, plus intelligible.

1. Corcoran 1972, 1973, 1974. 
gismes fondée sur l'explicitation d'une telle sémantique et sur la construction d'une preuve de complétude. On tient aujourd'hui pour acquis que la lecture des syllogismes comme règles $\mathrm{d}^{\text {'inférence }}{ }^{2}-$ et non comme propositions implicatives complexes, lecture suggérée, quant à elle, par Łukasiewicz - est la plus fidèle au texte des Analytiques, et c'est elle qui guide désormais les traductions et les commentaires, notamment ceux de Smith.

Dans ce récit du renouveau des études logiciennes d'Aristote, on passe sous silence une monographie parue en 1964 dans la série de langue allemande «Hypomnemata », discrètement présente dans de nombreuses bibliographies, à commencer par celle de la seconde édition de l'ouvrage de Patzig, mais presque jamais citée, intitulée Ein formales Modell der Syllogistik des Aristoteles et écrite par Kurt Ebbinghaus, un étudiant de P. Lorenzen. On peut le déplorer, car outre l'impression qui s'en dégage que l'interprétation des syllogismes comme règles d'inférence y est déjà contenue, cet opuscule présente un intérêt majeur pour comprendre ce qui fait d'Aristote un grand logicien et qui tient notamment à l'originalité de sa propre pensée de la « complétude », pensée qui peut être opposée à celle qui guide notamment J. Corcoran lorsqu'il entreprend d'établir, par une preuve, la complétude de la logique d'Aristote en relayant de ses propres efforts ceux d'Aristote lui-même ${ }^{3}$. Dans cet article, nous revenons d'abord sur les paradoxes de l'interprétation proposée par Łukasiewicz, afin de montrer en quoi l'interprétation d'Ebbinghaus adhère bien mieux au texte des Premiers Analytiques. Puis nous comparons le modèle formel proposé par ce dernier au modèle mathématique proposé par Corcoran, afin d'en exhiber la proximité. Pour finir, nous essayons de dégager de l'article d'Ebbinghaus une conception de la « complétude » que nous opposons à la « preuve de complétude » que Corcoran s'est efforcé de greffer au système de déduction naturelle qu'il attribue à Aristote.

2. En fait, cette « nouvelle » manière de voir le syllogisme est souvent appelée aujourd'hui, à juste titre, la perspective de « Corcoran-Smiley ». En effet, bien que le système de déduction naturelle développé par chacun d'eux diffère (voir Smiley 1962, 1973), ils partagent le même type de système formel, notamment un système de déduction naturelle associé à une sémantique formelle sous-jacente (ainsi qu'une critique de la reconstruction de Łukasiewicz). Nous avons focalisé notre discussion sur le modèle de Corcoran, afin de disposer d'un terme de comparaison bien défini ; mais rien dans notre propos ne repose sur le fait d'avoir choisi Corcoran plutôt que Smiley. D'ailleurs, la reconstruction de Smiley 1962 basée sur une « sémantique avec domaines triés » (many-sorted semantics) apparaît plus adéquate que celle utilisée par Corcoran 1972. Cependant, l'article en question concerne la notion d'ecthèse, qui n'est pas le sujet d'Ebbinghaus (bien que dans une note de bas de page, ce dernier suggère la possibilité de rendre compte de l'ecthèse au moyen d'une logique dialogique) et n'a pas de lien avec la preuve de complétude de Corcoran 1972. On dispose aujourd'hui de plusieurs reconstructions alternatives sophistiquées qui suivent une approche en termes de déduction naturelle. L'une des premières est celle de Thom 1981.

3. Corcoran 1972. 


\section{Les limites de la reconstruction de Eukasiewicz}

Dans son ouvrage, traduit en français sous le titre La syllogistique d'Aristote dans la perspective de la logique formelle moderne, tukasiewicz fait un travail de pionnier et cherche à instaurer un dialogue avec Aristote en le défendant à la fois contre l'interprétation traditionnelle et contre les reproches qui lui sont faits par la logique moderne $e^{4}$. Ce travail est d'abord un travail de retour au texte, même s'il rencontre des limites considérables.

Pour présenter tout d'abord les grandes lignes de cette reconstruction, on peut énoncer les points suivants :

- Łukasiewicz estime tout d'abord qu'Aristote n'est pas rigoureux dans son formalisme et qu'il joue sur les correspondances intuitives propres au langage naturel. Il y aurait formalisme si à ce « jeu » étaient substituées des formes parfaitement univoques, ce que Łukasiewicz propose de faire.

- Un syllogisme ne représente pas, selon Łukasiewicz, une règle d'inférence, mais une proposition du type «si $\mathrm{p}$ et $\mathrm{q}$, alors $\mathrm{r} »$, c'està-dire une proposition exprimant une implication matérielle. Pour affirmer cela, il s'appuie sur la manière dont Aristote formule explicitement ses syllogismes. Par exemple, « Si A s'applique à tout $\mathrm{B}$ et $\mathrm{B}$ à tout $\mathrm{C}$, alors il est nécessaire que $\mathrm{A}$ s'applique à tout $\mathrm{C} \gg$ (APr. 1, 4, 25b38). En outre, un syllogisme ne met pas en relation des propositions, mais des termes.

- La proposition qu'est le syllogisme est non quantifiéé. Les quantificateurs apparents que sont tout, aucun, quelque ne sont que des parties des constantes $\mathrm{A}$, E, I et $\mathrm{O}$, qui représentent des types de relations entre des termes. Il y a toutefois une quantification universelle implicite dans toutes les relations entre termes définies par Aristote. C'est cette quantification universelle qui constitue la nécessité syllogistique.

- Enfin, les syllogismes parfaits sont, selon Łukasiewicz, des axiomes dont vont être dérivés les syllogismes imparfaits au moyen de lois qu'Aristote utilise intuitivement, mais qu'il n'explicite pas et qui correspondent aux lois du calcul des propositions. Il y a donc là aussi une dimension implicite dans les raisonnements d'Aristote. Son travail de logicien est alors incomplet et Łukasiewicz se propose de le compléter ${ }^{6}$, au moyen des outils de la logique moderne.

4. Voir Gourinat 2011.

5. « Aristote ne s'est pas formé une idée très claire des quantificateurs et n'en a pas fait usage dans ses œuvres ».

6. « Il est impossible de comprendre complètement les démonstrations d'Aristote si l'on méconnaît l'existence d'un autre système que le sien, qui lui est parallèle tout en étant plus fondamental que la théorie du syllogisme. Il s'agit de la logique des propositions » (p. 65). 
À partir de l'ensemble de ces choix théoriques, Łukasiewicz parvient à restituer l'ensemble des résultats obtenus par Aristote, mais c'est au prix d'un décalage avec le texte et les procédés effectivement mis en œuvre par Aristote.

On peut noter les points suivants :

- Si les syllogismes étaient effectivement des propositions, alors Aristote devrait considérer que les syllogismes valides sont des propositions vraies. Or, à aucun moment il ne qualifie un syllogisme de « vrai ».

- Si les syllogismes étaient des propositions conditionnelles, alors on ne pourrait pas comprendre pourquoi Aristote soutient qu'une démonstration est un syllogisme ${ }^{7}$. Une démonstration ne peut évidemment pas consister en une seule proposition.

- L'idée qu'un syllogisme est une proposition conduit Łukasiewicz à rejeter la manière dont Aristote s'y prend explicitement pour réduire les syllogismes imparfaits aux syllogismes parfaits, ou du moins pour substituer à un procédé déductif encore trop intuitif une expression rigoureusement formaliste. Considérons en guise d'exemple deux des trois types de preuve proposés par Aristote (la preuve par conversion et la preuve par l'impossible), afin de montrer comment Łukasiewicz s'y prend pour les reconstruire.

La preuve par conversion (Festino, mode de la deuxième figure)

Voici le texte d'Aristote :

Si M n'est le cas pour aucun $\mathrm{N}$ et est le cas pour tel $\mathrm{P}$, alors, nécessairement $\mathrm{N}$ n'est pas le cas pour tel P. Car puisque la négative se convertit, $\mathrm{N}$ n'est le cas pour aucun $\mathrm{M}$. Or, on avait supposé que $\mathrm{M}$ était le cas pour tel $\mathrm{P}$, de sorte que $\mathrm{N}$ ne sera pas le cas pour tel $\mathrm{P}$ (en effet, on a ici une déduction de la première figure). ( $A P r$. I, 5, 27a32-36 ${ }^{8}$ )

Selon Łukasiewicz, « Aristote effectue [là] une preuve intuitive », dont, selon lui, l'analyse révèle qu'elle contient implicitement deux thèses du calcul propositionnel, qu'il va, quant à lui, expliciter.

La première énonce :

$$
(p \rightarrow q) \rightarrow((p \wedge r) \rightarrow(q \wedge r))
$$

Si on définit $p: \mathrm{M}$ n'est le cas d'aucun $\mathrm{N} ; q: \mathrm{N}$ n'est le cas d'aucun $\mathrm{M} ; r: \mathrm{M}$ est le cas de quelque $\mathrm{P}$, alors l'antécédent correspond à la loi de conversion, admise par Łukasiewicz dans sa reconstruction axiomatique. On peut donc détacher le conséquent, à savoir : « Si M n'est le cas d'aucun N et si M est le cas de quelque $\mathrm{P}$, alors $\mathrm{N}$ n'est le cas d'aucun $\mathrm{M}$ et $\mathrm{M}$ est le cas de quelque $\mathrm{P} \gg$.

Si maintenant on définit $p: M$ n'est le cas d'aucun $\mathrm{N}$ et $\mathrm{M}$ est le cas de quelque $\mathrm{P} ; q: \mathrm{N}$ n'est le cas d'aucun $\mathrm{M}$ et $\mathrm{M}$ est le cas de quelque $\mathrm{P}$; et $r$ :

7. $A P r . \mathrm{I}, 4,25 \mathrm{~b} 30$.

8. Traduction de M. Crubellier 2014. 
$\mathrm{N}$ n'est pas le cas de quelque $\mathrm{P}$, alors en invoquant la seconde thèse du calcul propositionnel, qui énonce que :

$$
(p \rightarrow q) \rightarrow((q \rightarrow r) \rightarrow(p \rightarrow r))
$$

et en s'appuyant sur le conséquent détaché auparavant (ici $p \rightarrow q$ ), on établit que si on admet le mode Ferio, c'est-à-dire : « Si N n'est le cas d'aucun M et si $\mathrm{M}$ est le cas de quelque $\mathrm{P}$, alors $\mathrm{N}$ n'est pas le cas de quelque $\mathrm{P} \gg$ (ici : $q \rightarrow r$ ), alors on est obligé d'admettre le mode Festino, c'est-à-dire : « Si M n'est le cas d'aucun $\mathrm{N}$ et $\mathrm{M}$ est le cas de quelque $\mathrm{P}$, alors $\mathrm{N}$ n'est pas le cas de quelque $\mathrm{P} \gg($ ici $: p \rightarrow r)$.

On constate que l'analyse que Łukasiewicz propose de la démonstration « intuitive »d'Aristote distingue des rapports logiques qui n'apparaissent pas à Aristote, mais sur lesquels celui-ci s'appuie « sans le savoir ${ }^{9}$.

\section{La preuve par l'impossible (Baroco)}

Aristote argumente ainsi :

Si maintenant $\mathrm{M}$ est le cas pour tout $\mathrm{N}$ et n'est pas le cas pour tel $\mathrm{P}$, nécessairement $\mathrm{N}$ n'est pas le cas pour tel $\mathrm{P}$; car s'il était le cas pour tout $\mathrm{P}$, étant donné que par ailleurs $M$ s'applique à tout $N$, il serait nécessaire que $M$ soit le cas pour tout $\mathrm{P}$; mais on a supposé qu'il n'était pas le cas pour tel $\mathrm{P}$. (APr. I, 5, 27a36-b1)

Selon Łukasiewicz, ce procédé ne peut pas constituer une vraie preuve par l'impossible du syllogisme, car il repose sur la négation non pas du syllogisme entier, mais seulement de la conclusion ( « N n'est pas le cas pour tel $\mathrm{P} »)$.

Łukasiewicz va donc là aussi proposer une toute autre démonstration, en s'appuyant sur la loi de la logique des propositions appelée « loi de transposition $\gg$ qui énonce :

$$
((p \wedge q) \rightarrow r) \rightarrow((p \wedge \neg r) \rightarrow \neg q) .
$$

Si on définit $p: \ll \mathrm{M}$ est le cas pour tout $\mathrm{N} \gg ; q:\langle\mathrm{N}$ est le cas pour tout $\mathrm{P} \gg$ et $r$ : « $\mathrm{M}$ est le cas pour tout $\mathrm{P} »$, alors, d'après la loi de transposition, l'antécédent correspondant au syllogisme parfait Barbara donne bien le syllogisme de la deuxième figure Baroco.

Selon Łukasiewicz, les démonstrations proposées par Aristote sont donc menées dans une certaine inconscience, car elles comportent une dimension intuitive à laquelle on peut substituer une expression formaliste plus rigoureuse. Aristote ne serait, en somme, pas vraiment conscient de ce qu'il fait : non conscient que les syllogismes parfaits sont des axiomes, ni que les démonstrations qu'il effectue ne sont pas des syllogismes, ni que les fondements d'une preuve par conversion sont plus complexes qu'il ne le croit, ni que les preuves qu'il propose comme étant des preuves par l'impossible ne

9. Ou peut-être plutôt sans s'y intéresser. Il ne serait pas illégitime de penser que les dérivations formulées par Łukasiewicz dans le calcul des propositions correspondent plutôt à des métarègles, secondaires par rapport à la marche naturelle des raisonnements. 
satisfont pas aux exigences d'une véritable preuve par l'impossible. Il apparaît donc que la lecture proposée par Łukasiewicz est peu charitable. Elle conduit à croire que le travail d'Aristote est plutôt un travail d'éclaireur, comme si Aristote formulait des conjectures sans les accompagner des outils d'analyse adéquats. Aristote serait donc, dans les textes des Analytiques, dans un rapport d'approximation avec des vérités logiques qu'il devine plus qu'il ne les conceptualise adéquatement. Łukasiewicz conçoit alors son propre travail comme comblant, au moyen des outils de la logique moderne, les lacunes et les carences du travail d'Aristote. Selon ce point de vue, le développement historique de la logique pourrait donc être mesuré à l'aune de sa finesse expressive. Les efforts d'Aristote peuvent être vus comme encore un peu maladroits, si on les évalue à l'aune des résultats postérieurs de la logique.

\section{Le modèle formel d'Ebbinghaus et sa proximité avec le texte des Analytiques}

Dans un effort d'adhérence minutieuse à la lettre du texte, Ebbinghaus va paradoxalement concevoir son modèle à travers ce qu'il appelle un «calcul » ${ }^{10}$, c'est-à-dire ici un ensemble de règles qu'on peut faire correspondre à des schémas opératifs. En ce sens très large du mot « calcul », tricoter est un calcul : quelqu'un qui tricote applique une règle implicite qui lui permet de répéter des opérations. On peut dire qu'un calcul n'est rien d'autre que l'explicitation par des règles d'une méthode de construction de quelque chose à partir de quelque chose. Les syllogismes correspondent ici à des règles de ce type. Selon cette approche, la signification des expressions qui apparaissent dans un syllogisme est donnée par les propriétés inférentielles prescrites par les règles opératives.

Pour être exact, Ebbinghaus va en fait reconstruire la théorie du syllogisme à partir de deux calculs. Le premier prend pour «input » des phrases concrètes et les transforme en des formes propositionnelles de 4 types: $A a B$, $\mathrm{CeD}, \mathrm{EiF}, \mathrm{GoH}$. Les « points de départs » $(\text { Anfänge })^{11}$ d'un raisonnement sont déjà les résultats de l'application d'opérations schématiques. À partir

10. Ce concept particulier de « calcul », compris comme un ensemble d'opérations schématiques à partir desquelles on se donne le droit de produire des signes déterminés à partir de signes donnés, est tiré, d'ailleurs comme la plupart des concepts sur lesquels s'appuie Ebbinghaus, de l'ouvrage de Lorenzen, Einführung in die operative Logik und Mathematik, 1959.

11. Dans la traduction de la monographie d'Ebbinghaus par C. Lion, le choix a été fait de traduire «Anfänge » par « points de départ » plutôt que par « formules initiales », afin de souligner le fait qu'un calcul conçu selon la méthode opérative ne relève pas à proprement parler d'un « langage », possédant une syntaxe qui aurait besoin d'être interprétée, mais ne consiste qu'en de pures règles opératoires. Lorsque Ebbinghaus ajoute une prémisse au calcul, afin de prouver, par l'absurde, la contradictoire de cette prémisse, nous avons utilisé « formule initiale ajoutée », ce qui dans un vocabulaire plus moderne correspondrait à « hypothèse locale » (voir Un modèle formel de la Syllogistique d'Aristote, trad. C. Lion 2016). Cela étant, 
d'un ensemble infini de phrases, on peut construire quatre ensembles correspondant aux propositions de type $A, E, I$ et $O$. À la base de la théorie d'Aristote, il y aurait un acte de construction de prémisses possibles. Ce point permet d'expliciter la règle qu'Aristote emploie implicitement lorsqu'il désigne une même proposition sous plusieurs formes différentes, par exemple « $\mathrm{A}$ est prédiqué de tout $\mathrm{B}$ » ou « $\mathrm{A}$ appartient à tout $\mathrm{B}$ ». Cet usage indifférencié de formules équivalentes correspond à une opération, à une sorte de fonction injective, qui à un ensemble de phrases fait correspondre une seule et unique forme abstraite. Ce calcul auxiliaire qu'Ebbinghaus situe en amont de son modèle semble correspondre à une opération constructive qui situe d'emblée l'activité proprement logique dans un domaine d'objets qui est fabriqué à partir des discours réels, afin de permettre la réalisation d'opérations plus complexes qui seront effectuées dans le calcul proprement dit. Le fait qu'Aristote emploie indifféremment des formules équivalentes ne signifie donc pas un défaut de formalisme, mais plutôt l'indication que quelque chose est à construire à partir de ces formules diverses, à savoir une forme schématique.

Ce calcul auxiliaire (nommé $K_{s}^{*}$ ) étant posé, Ebbinghaus va en définir un second, qu'il nomme $K_{s}$ et qui consiste lui aussi en un ensemble de règles de construction à appliquer aux constructions qui résultent elles-mêmes du calcul auxiliaire. Ce calcul est le suivant :

$$
\begin{aligned}
& \left(R_{1}\right) \quad A e B \rightarrow B e A \\
& \left(R_{2}\right) \quad A a B, B a C \rightarrow A a C \text { (Barbara) } \\
& \left(R_{3}\right) \quad A e B, B a C \rightarrow A e C \text { (Celarent) } \\
& \left(D_{l}\right) \quad A a B, A o B \rightarrow \Lambda \\
& \left(D_{2}\right) \quad A e B, A i B \rightarrow \Lambda \\
& \left(D_{3}\right) \quad A a B, A e B \rightarrow \Lambda \\
& \left(R_{4.1}^{3}\right) A a B \rightarrow \Lambda \dot{\rightarrow} A o B \\
& \left(R_{4.2}^{4.1}\right) A e B \rightarrow \Lambda \dot{\rightarrow} A i B \\
& \left(R_{4.3}^{4.2}\right) \quad A i B \rightarrow \Lambda \rightarrow \dot{A} A e B \\
& \left(R_{4.4}^{4.3}\right) A o B \rightarrow \Lambda \rightarrow \\
& \left(R_{5}\right) \quad A a B, B i C \rightarrow A i C \text { (Darii) } \\
& \left(R_{6}\right) \quad A e B, B i C \rightarrow A o C^{12} \text { (Ferio) }
\end{aligned}
$$

Bien que ces règles constituent un calcul autonome, on peut les faire correspondre aux différents types de règles dont Aristote fait un usage explicite au début des Premiers Analytiques. Il est tout d'abord important de

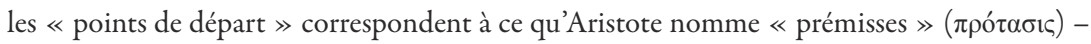
« hypothèse globale ».

12. Ces deux dernières règles peuvent être mises à part, car Ebbinghaus montrera qu'elles sont « admissibles », c'est-à-dire qu'elles correspondent à des syllogismes qu'il est possible de réduire aux syllogismes parfaits auxquels correspondent les règles $R_{2}$ et $R_{3}$, ce que fait également Aristote au chapitre 7 du livre 1 des Premiers analytiques. 
souligner que les opérateurs $a, e, i$ et $o$ correspondent respectivement, dans le texte d'Aristote, aux types de prémisse : « ...est prédiqué de tout... », «...n'est prédiqué d'aucun... », « ...est prédiqué de quelque... », « ...n'est pas prédiqué de quelque... ». Ceci étant posé, la règle $\left(R_{l}\right)$ correspond à une régle de conversion du sujet et du prédicat dans une prémisse du type $\ll \ldots$ n'est prédiqué d'aucun... ». Il est important de garder à l'esprit qu'Ebbinghaus définit ici les régles nécessaires et suffisantes pour la signification de la conversion dans le contexte de $K_{s}^{*}$ : la validité de la conversion du sujet et du prédicat dans une universelle négative ne requiert pas l'ajout au système d'une définition vérifonctionnelle.

On peut en dire autant des règles qui suivent. $\left(R_{2}\right)$ et $\left(R_{3}\right)$ correspondent aux règles des syllogismes parfaits (Barbara et Celarent). $\left(D_{1}\right),\left(D_{2}\right)$ et $\left(D_{3}\right)$

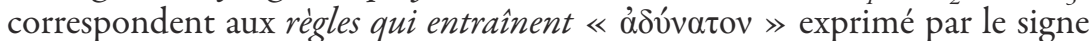
$\wedge^{13}$ quand sont prises comme points de départ les formules antécédentes des règles de type $D$. Ces règles correspondent en fait, pour $D_{1}$ et $D_{2}{ }^{14}$, à la


règles $\left(R_{4.1}\right),\left(R_{4.2}\right),\left(R_{4.3}\right)$ et $\left(R_{4.4}\right)$ sont les régles de négation, qui sont décisives en vue de la reconstruction des preuves indirectes (qu'Ebbinghaus nomme «syllogismi per impossibile ») $)^{15}$. Ebbinghaus note à leur propos qu'elles sont des métarègles, c'est-à-dire qu'elles ne font pas qu'ajouter au calcul des figures dérivables, mais contiennent aussi - dans leurs formules antécédentes - une phrase à propos du calcul, à savoir une affirmation de dérivabilité, du type : « Si du fait de l'ajout de $A a B$ au calcul, $\wedge$ devient dérivable, alors... ». La formule antécédente appartient ainsi à un niveau supérieur, étant donné qu'elle désigne non pas une figure dérivée, mais une opération de dérivation (p. 25 de la traduction française).

Pour marquer le contraste avec la perspective de Łukasiewicz, on peut noter les points suivants, à propos du calcul $K_{\mathrm{s}}$ :

- Les syllogismes correspondent bien ici à des règles d'inférence, c'est-à-dire à des schémas opératifs, tels qu'à partir de quelque chose de donné, on peut construire quelque chose d'autre. La flèche désigne donc une action de construction de quelque chose à partir de quelque chose d'autre. La question de la vérité n'a pas du tout à être posée dans ce cadre ; du moins, elle n'a pas à être posée pour

13. Cf. Ebbinghaus 2016.

14. Mentionnons ici que Eukasiewicz avait déjà observé (p. 56) que les universelles négatives ne sont rien d'autre que la négation des universelles affirmatives.

15. Pour une explication plus exhaustive de ce à quoi correspondent les règles de Ks dans les Premiers Analytiques, on se reportera au chapitre $2 \$ 5$, intitulé « Preuve de l'isomorphisme » dans lequel Ebbinghaus montre, à travers une attention très scrupuleuse au texte d'Aristote, les correspondances qu'on peut établir avec son propre calcul. 
comprendre la nature du syllogisme $e^{16}$.

- Si on parvient à établir que les démonstrations proposées par Aristote pour réduire les syllogismes correspondent bien à une application de ces mêmes syllogismes, alors Aristote ne fait pas erreur quand il affirme que les démonstrations sont des syllogismes.

- Les procédés de réduction d'un syllogisme à un autre ne correspondent pas, dans ce cadre, à la démonstration d'un théorème à partir d'axiomes, mais plutôt à la démonstration qu'une règle est réductible à d'autres. Ebbinghaus appelle admissibilité (terme repris à Lorenzen) le statut d'une règle qui permet de construire quelque chose qui pourrait très bien être construit sans cette règle, qui constitue donc une sorte de détour ${ }^{17}$. Les syllogismes imparfaits sont réductibles aux syllogismes parfaits, car ce qu' ils permettent de construire peut très bien être construit sans eux ; autrement dit, ils n'ajoutent rien à la capacité proprement opérative procurée par les règles fondamentales du calcul, sinon une simplification des procédures. Nous reviendrons plus loin sur le concept d'admissibilité, lorsque nous aborderons la question de la complétude.

- Enfin, le calcul $K_{s}$ offre un moyen élégant de rendre compte des preuves indirectes. Nous l'illustrerons juste après (exemple 2). Notons tout de suite qu'elles sont effectuées par Ebbinghaus par le biais des règles $\left(D_{1}\right),\left(D_{2}\right),\left(D_{3}\right),\left(R_{4.1}\right),\left(R_{4.2}\right),\left(R_{4.3}\right)$ et $\left(R_{4.4}\right)$, c'est-àdire d'une manière purement inférentielle et selon la procédure qui suit. Pour développer une preuve indirecte :

1. On ajoute, à l'ensemble initial de prémisses, la négation de la conclusion (dans l'exemple proposé plus bas $\mathrm{NaP}$ ).

2. En se servant des règles de type $R$ (dans l'exemple $2\left(R_{2}\right)$ = Barbara) et des règles de type $D$, on montre que cet ajout conduit à une contradiction avec l'une des prémisses (dans l'exemple $2 \mathrm{MoP}$ ).

3. En appliquant alors l'une des règles de type $R$ (dans l'exemple $\left.2\left(R_{4.1}\right)\right)$, on obtient la négation de la prémisse ajoutée, c'est-à-

16. « La flèche, ici équivalente au "si...alors" aristotélicien, n'est pas [...] définie à travers la valeur de vérité, mais elle exprime une indication d'action. Nous nous écartons par conséquent des interprétations qui jusqu'ici ont considéré le "si...alors" aristotélicien en tant qu' implication, au sens de l'implication matérielle et formelle (Łukasiewicz 1951 ; Bocheński 1951 ; Patzig 1959) », Ebbinghaus 2016 p. 28.

17. Ou «peak» (Gentzen 1969). On peut prouver l'admissibilité d'une règle au moyen d'une « méthode d'élimination » (Lorenzen) - qu'on appelle plutôt « réduction des détours » en théorie de la preuve - elle-même fondée sur le concept de « dérivabilité » : une règle est admissible, si l'élimination de cette règle maintient la dérivabilité dans le calcul de toutes les formules dérivables dans le calcul complété au moyen de cette règle. Sur la méthode de l'élimination, voir Lorenzen $19691 \$ 3$. 
dire la négation de la négation de la conclusion.

4. On obtient alors, si l'on suppose la double négation (comme c'est bien le cas lorsqu'on procède par l'absurde), la preuve de la conclusion visée $\mathrm{e}^{18}$.

Reprenons donc, à présent, les preuves par conversion et par l'impossible dont nous avons précédemment montré en quoi consistait leur reconstruction formaliste chez Łukasiewicz ${ }^{19}$, afin de voir, en contrepoids, comment Ebbinghaus les restitue dans son propre modèle formel, sous la forme de preuves d'admissibilité.

\section{Exemple 1}

Pour la preuve par conversion (preuve directe), dans $K$, le mode Festino correspond à la règle :

$$
\mathrm{MeN}, \mathrm{MiP} \rightarrow \mathrm{NoP}
$$

Une dérivation contenant cette règle a la forme :

$$
\begin{array}{cc} 
& \\
l & \\
\vdots & \\
m e N \\
\\
\quad m & M i P \\
l, m \rightarrow n & N o P
\end{array}
$$

On peut remplacer cette dernière ligne de la façon suivante :

$$
\begin{array}{lll}
l \rightarrow n & \text { NeM } & \left(R_{l}\right) \\
n, m \rightarrow n+1 & \text { NoP } & \left(R_{6}\right)
\end{array}
$$

À la ligne $n$ correspond chez Aristote : « Étant donné que la négation peut être inversée $\left(R_{1}\right), N$ n'est le cas d'aucun $M$. » À la ligne $m:$ On a posé au départ que $M$ est le cas de quelque $P$ ». À la ligne $n+1:$ Ainsi, $N$ n'est pas le cas de quelque $P \gg$. Finalement, Aristote indique à propos de la règle employée $\left(R_{6}\right)$ : «Par conséquent, cela est produit à travers $(\delta 1 \alpha ́)$ un syllo-

18. « Aristote nomme explicitement (par exemple, APr. I, 23, 41a36) [la méthode de

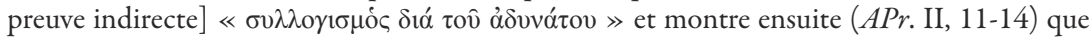
chaque phrase qu'on peut prouver par ce moyen est aussi démontrable au moyen d'un syllogisme direct. Il décrit le syllogismus per impossibile dans plusieurs passages (APr. I, 23 ; I, 44; II, 11-14), mais ne le formule jamais sous la forme d'un énoncé complet du type si-alors. Il propose toujours plutôt une description plus ou moins compliquée d'un procédé, dont il apparaît qu'à chaque fois, ce qu'il en dit est isomorphe à la règle $R_{4} d u$ calcul $K_{\text {s }}$ Les formules antécédentes correspondent par ailleurs à des syllogismes directs. » Ebbinghaus 2016 p. 31 (nous soulignons).

19. Ebbinghaus déclare ne pas disposer avec $K_{s}$ d'un moyen adéquat de reconstruire la preuve par ecthèse. Cependant, nous évoquerons à nouveau ce point un peu plus loin, lorsque nous reviendrons sur la thèse de Eukasiewicz selon laquelle il n'y aurait aucune théorie des quantificateurs chez Aristote. 
gisme de la première figure $\gg$.

Exemple 2

Pour ce qui concerne maintenant la preuve indirecte, dans $K_{s}$ Baroco correspond à la règle :

$$
M a N, M o P \rightarrow N o P
$$

Sa dérivation correspond au schéma suivant :

$\begin{array}{clc}\vdots & & \\ l & M a N & \\ \vdots & M o P & \\ \vdots & & \\ n & N a P & \text { (formule initiale ajoutée) } \\ l, n \rightarrow n+1 & M a P & \left(R_{2}\right) \\ n+1, m \rightarrow n+2 & \wedge & \left(D_{1}\right) \\ n \rightarrow n+2 \rightarrow-n+3 & N o P & \left(R_{4,1}\right)\end{array}$

On peut comparer cette inférence avec le texte d'Aristote (pour le début, voir l'extrait d'Aristote retranscrit p. 5) : ligne $n:$ « Car s'il est le cas pour chaque $P \gg$, ligne $l: \ll$ et si $M$ est aussi dit de chaque $N \gg$, ligne $n+1: \ll$ il est nécessaire que $M$ soit le cas de chaque $P$. »; ligne $m:$ 《ais il était posé au départ que $M$ n'est pas le cas de quelque $P \gg$. Les lignes $n+2$ et $n+3$ sont à compléter : « Mais il est impossible que $M$ soit le cas de chaque $P$ et qu' il ne soit pas le cas pour quelque $P$. » $(n+2):$ « Il doit donc être faux que $N$ soit le cas de tout $P$, et par suite il n'est le cas d'aucun $\gg(n+3)$.

On constate que la fidélité au texte d'Aristote est dans les deux cas très exacte. La démonstration consiste à exhiber les opérations de construction qu'il est possible d'accomplir au moyen des seules règles initiales du calcul et dont les règles admissibles ne constituent qu'une explicitation.

Si l'on suit cette reconstruction, il apparaît qu'Aristote nomme les éléments de ses propres raisonnements avec une parfaite adéquation. Le fait qu'il n'emploie ni le concept d'axiome, ni celui de vérité dans le texte des Premiers analytiques apparaît parfaitement justifié. En somme, il est légitime de dire que la théorie du syllogisme expose les règles qu'Aristote emploie lorsqu'il établit des rapports de réduction entre les syllogismes. Ceci tend à prouver que les raisonnements effectués par Aristote ne présupposent aucune logique sous-jacente, mais constituent plutôt une exécution de la logique dont il définit lui-même les règles. Ainsi, tout se passe comme si Aristote commençait par formuler des règles, pour ensuite faire de ces règles des objets d'étude, à travers une sorte de procédé réflexif. Son travail de logicien ne s'arrête pas au seul acte de répertorier des formes valides de raisonnement, car il en vient à construire des relations entre ces mêmes formes, telles qu'il est possible d'établir que les unes n'augmentent nullement la puissance opérative des 
formes canoniques que constituent les syllogismes parfaits et la règle d'inversion. Cette démarche pourrait être qualifiée de métalogique si l'on entend par là non pas l'acte de formaliser les rapports entre des assertions, mais celui de formaliser les schémas d'inférence eux-mêmes pour construire des relations d'équivalence entre leurs diverses formes possibles, qui sont autant d'instruments permettant de décider de la validité d'un raisonnement.

Il est intéressant de remarquer qu'Ebbinghaus situe par ailleurs la théorie du syllogisme dans son contexte historique et qu' il justifie ainsi la pertinence de son approche opérative. Selon lui, l'origine du syllogisme est à rechercher dans les jeux dialectiques. À partir d'un certain niveau de codification de ces jeux, le besoin s'est fait ressentir de déterminer précisément ce qu'il convenait de reconnaître comme devant être éprouvé comme évident. Dans les Topiques, Aristote recommande de ne pas formuler en tant que question l'acte même de tirer la conclusion (158a7-13). En effet, si l'on pose cette question, le partenaire considèrera qu'il est possible de contester la légitimité de la conclusion. Cette solution comporte à l'évidence une dimension purement rhétorique, qui ne la rend pas pleinement satisfaisante. Néanmoins le problème est bien posé : comment contraindre un partenaire à tirer une conclusion ? Les Analytiques offrent une solution pour rendre parfaitement décidable la question de la validité ou non-validité de chaque étape de la discussion. Cela signifie que les Analytiques reprennent d'une manière systématique une réflexion qui s'impose naturellement aux partenaires d'un jeu dialectique ${ }^{20}$, et qui vise à dégager les moyens d'obtenir un accord sur

20. Cette manière de situer le sens de la théorie du syllogisme dans le contexte des jeux dialectiques ouvre d'ailleurs une perspective de recherche sur la question des quantificateurs chez Aristote. Souvenons-nous que pour Łukasiewicz, la théorie du syllogisme est une théorie dont toute quantification explicite est absente. Or, dans une note de bas de page, Ebbinghaus suggère qu'il est possible de dégager de textes extraits des Topiques, une théorie de la quantification des prémisses. Les quantifications universelles et existentielles vont être situées dans le contexte d'une interaction entre un proposant et un opposant sous la forme d'une procédure d'argumentation. Citons Ebbinghaus : « 1 . Si le proposant soutient une phrase de la forme "A est le cas de quelque B" et si l'opposant la nie, alors le proposant a gagné s'il parvient à proposer un cas n pour lequel les deux propositions primitives "A est le cas pour n" et "B est le cas pour n" sont vraies. S'il n'y parvient pas, alors il a perdu. 2. Si le proposant soutient une phrase de la forme "A est le cas de tout B" et si l'opposant la nie, alors le proposant doit convaincre l'opposant de la validité générale de son assertion en donnant plusieurs cas pour lesquels $\mathrm{A}$ et $\mathrm{B}$ sont valables. Mais comme l'assertion générale se rapporte à une classe infinie, l'opposant peut toujours nier celle-ci. Or, le proposant a le droit d'exiger de lui un contre-cas, c'est-à-dire que l'opposant doit donner un $\mathrm{n}$, pour lequel $\mathrm{n} \varepsilon \mathrm{B}$ soit valable, mais pas $\mathrm{n} \varepsilon \mathrm{A}$. S' $\mathrm{il}$ n'y parvient pas, alors le proposant a gagné et vice versa. (On trouve cette dernière règle explicitement dans les Topiques $\theta, 157 \mathrm{a} 34$; on nomme ce procédé l'induction, غ̇ं $\alpha \gamma \omega \gamma \eta ́)$ ». Ceci tend à établir qu'Aristote avait en sa possession une théorie de la quantification qui le rendait capable de distinguer entre une procédure argumentative fondée sur l'incapacité de l'opposant à fournir un contre-exemple (dans le cas d'une proposition universelle) et sur la capacité du proposant à fournir un exemple (dans le cas de la proposition particulière). Il convient de 
les règles d'inférence. La théorie du syllogisme répondrait donc à un besoin immanent aux jeux dialectiques. Elle doit donc être fondée en dernière instance sur une perspective pragmatique et dialogique.

\section{Le modèle de J. Corcoran, sa proximité avec le modèle d'Ebbinghaus}

Si l'on se cantonnait à une compréhension de la sémantique en termes de théorie des modèles, on pourrait être étonné de l'absence apparente de toute considération de cet ordre dans l'article d'Ebbinghaus et croire que la validité des règles d'inférence que sont les syllogismes demanderait à être fondée dans une discipline formelle remplaçant l'ontologie, dont Ebbinghaus concède qu'elle complète, chez Aristote, les recherches proprement logiques ${ }^{21}$. On serait alors tenté de penser que le modèle d'Ebbinghaus est incomplet, car le système de règles d'inférence qu'il propose n'est associé à aucune « fonction de vérité », ni à aucun « univers de discours ». On chercherait pourtant en vain des formules indiquant qu'Aristote lui-même envisage comme pertinente la perspective d'un langage non interprété. John Corcoran le concèdera dans l'article « Aristotle's natural deduction system » : «le concept [d'un univers de discours] ne joue aucun rôle dans le système des Premiers analytiques $\gg$ (p. 104). Mais s'il le concède, c'est parce que dans son propre modèle, il fondera les règles d'inférence que constituent, pour Ebbinghaus comme pour lui, les syllogismes, sur un système sémantique basé sur des notions extensionnelles, et donc sur la possibilité théorique de concevoir des univers de discours alternatifs ${ }^{22}$, afin que soit universellement fondée la relation de conséquence logique. Avant d'étudier en quoi cette introduction d'un système sémantique dans le modèle que propose Corcoran l'éloigne de la lettre du texte des Analytiques, considérons d'abord son modèle en tant

remarquer que cette théorie de la quantification ne repose pas sur la mise en relation d'une proposition avec un domaine d'objets correspondant, mais bien sur une interaction au sein d'une dynamique argumentative qui repose sur une prise en compte de la capacité des agents épistémiques à produire ou pas des exemples ou des contre-exemples. Notons au passage que s'il est vrai que cette approche dialogique permet de contester la thèse de Łukasiewicz selon laquelle on ne trouve aucune théorie de la quantification chez Aristote, du moins elle ne s'imbrique pas d'elle-même dans l'approche opérative qui est celle d'Ebbinghaus dans la construction du calcul $K_{s}$. Ajoutons enfin qu'Ebbinghaus exprime à plusieurs reprises l'idée qu'il ne dispose pas d'une théorie des prémisses. Sur la quantification universelle et une première réhabilitation de l'importance de l'article d'Ebbinghaus, voir Marion \& Ruckert 2016. Sur une interprétation proprement dialogique de la théorie du syllogisme assertorique, voir Rahman, McConaughey \& Crubellier, en préparation.

21. «Si on aborde le système aristotélicien avec le concept moderne de la règle et si on se demande pourquoi cette règle est valide, alors on ne peut donner à cela comme fondement qu'un état de fait, qui se laisse percevoir au moyen du vôিৎ et qui, dans le cadre de la pensée d'Aristote, relève proprement du domaine de l'ontologie. » Ebbinghaus 2016 p. 61.

22. Même si Corcoran précise que ce système déductif sera associé à une seule interprétation, considérée comme interprétation naturelle. 
que système formel de déduction naturelle.

Nous pouvons réduire ce système, qu'on nomme $D$ (pour «Deductive System »), au minimum en adoptant la présentation suivante ${ }^{23}$ (et en normalisant les expressions pour faciliter la comparaison avec les reconstructions respectives de Łukasiewicz et d'Ebbinghaus) ${ }^{24}$.

1. L'alphabet de $D$ contient :

a) Un ensemble $V$ de termes constants (A, B, C, D...)

b) Les opérateurs du syllogisme $(a, e, i, o)$

2. Les expressions bien formées du langage $L$, pour $D^{25}$, sont :

a) Pour tous termes constants distincts ${ }^{26} \mathrm{~A}$ et $\mathrm{B}: \mathrm{AaB}, \mathrm{AeB}, \mathrm{AiB}$ et $\mathrm{AoB}{ }^{27}$ sont des expressions bien formées (ou des « phrases »).

b) Rien d'autre n'est une expression bien formée.

3. Les règles d'inférence pour $D$. Pour tout $\mathrm{x}, \mathrm{y}, \mathrm{z} \varepsilon \mathrm{V}$ :
C1. x e y
C2. $\mathrm{x}$ a $\mathrm{y} \vdash \mathrm{y}$ ix (conversion)
C3. $x$ i y $\vdash$ y ix (conversion)
PS1. $x$ a y, y a $z \vdash x$ a z (Barbara)

23. Voir Fillion 2007 à qui nous reprenons la présentation du système déductif $D$ notamment dans le choix de remplacer le signe $\vDash$ par le signe $\vdash$, afin de faire ressortir provisoirement la primauté des règles d'inférence sur les relations de conséquence logique, choix sur lequel nous reviendrons plus bas.

24. Ainsi, au lieu de Aab (pour tout a est b), on écrira BaA (B est le cas de tout A)

25. Notons que Corcoran distingue le système déductif $D$ du langage $L$, qui en constitue la partie purement syntaxique.

26. Cette stipulation que les termes doivent nécessairement être distincts dans les expressions bien formées permet à Corcoran d'exclure les prémisses du type « Tous les hommes sont hommes ». Notons, à cette occasion, que Smiley 1973 p.153 mentionne la monographie d'Ebbinghaus et sa différence par rapport à l'approche de Eukasiewicz. Cependant, selon lui, cette reconstruction procède bien d'une approche par la déduction naturelle et non par des propositions implicatives, mais elle est trop permissive, en ce qu'elle autorise la constitution de prémisses du type AaA. Sur ce point précis, il faut concéder que ce type de prémisse ne correspond pas à l'approche épistémique qui paraît bien être celle d'Aristote dans les Analytiques. Cela étant, Corcoran 1974 p. 99 mentionne lui-même deux occurrences d'un tel type de proposition, qui seraient exclues comme étant mal formées selon les règles qu'il a définies lui-même, à savoir (APr. 63b40-64b25) : « Aucun savoir n'est savoir » et « Quelque savoir n'est pas savoir ». Sans doute Corcoran a-t-il raison de dire que de telles propositions ont un caractère « extra-systématique » chez Aristote, mais après tout, la permissivité du système d'Ebbinghaus lui permet de rendre compte d'une forme propositionnelle dont l'usage est attesté chez Aristote. L'argument que Smiley utilise pour établir le caractère non aristotélicien du système d'Ebbinghaus ne saurait apparaître comme absolument décisif et pourrait très bien être retourné contre le système de Corcoran.

27. En fait, Corcoran utilise une autre notation. Au lieu des constantes traditionnelles a, e, i et o, il utilise A, N, S et \$, pour « all », «no », « some » et « some... not ». Cette différence de notation ne change évidemment rien. 




Définition 1 (argument) : Un argument est une paire ordonnée $(\mathrm{P}, \mathrm{c})$, où $P$ est un ensemble de phrases (appelées prémisses) et c est une seule phrase (appelée conclusion).

Définition 2 (déduction directe) : Une déduction directe dans $D$ de c à partir de $\mathrm{P}$ est une liste finie de phrases terminant par c, commençant par toutes ou plusieurs phrases de $\mathrm{P}$, et telle que toute phrase subséquente (après celles de P) est soit :

- Une répétition d'une ligne qui précède

- Une $D$-conversion d'une ligne précédente

- Une $D$-inférence de deux lignes précédentes.

Définition 3 (déduction indirecte) : Une déduction indirecte dans $D$ de c à partir de $\mathrm{P}$ est définie comme une liste finie de phrases finissant par une paire de contradictoires $[\mathrm{d}, \mathrm{C}(\mathrm{d})]$, commençant par une liste de toutes ou plusieurs phrases de $\mathrm{P}$ suivies de $\mathrm{C}(\mathrm{c})$, et telle que chaque ligne additionnelle subséquente (après $\mathrm{C}(\mathrm{c})$ ) est soit :

- Une répétition d'une ligne qui précède

- Une $D$-conversion d'une ligne précédente.

- Une $D$-inférence de deux lignes précédentes.

En nous appuyant sur ce système déductif, montrons à présent comment on peut réduire les syllogismes Festino et Baroco. Il ne s'agit pas des exemples que prend Corcoran, mais d'exemples reconstruits selon les règles de son système. Pour rendre plus facile la comparaison de ces reconstructions avec celles proposées par Ebbinghaus, on se reportera aux tableaux proposés en annexe

\begin{tabular}{lll}
\multicolumn{3}{c}{ Festino (déduction directe) } \\
1 & $\mathrm{MeN}$ & Prémisse \\
2 & $\mathrm{MiP}$ & Prémisse \\
3 & $\mathrm{NeM}$ & $\mathrm{C} 1(1)$ \\
4 & $\mathrm{MiP}$ & Réitération $(2)$ \\
5 & $\mathrm{NoP}$ & PS4 $(3,4)$
\end{tabular}

28. $\mathrm{x}$ a $\mathrm{y}$ et $\mathrm{x}$ e $\mathrm{y}$ sont définis respectivement comme les contradictoires de $\mathrm{x}$ o $\mathrm{y}$ et de $\mathrm{x}$ i $\mathrm{y}$ (et vice versa). C(d) indique le contradictoire de d. CTR pour « contradiction ». 


\begin{tabular}{|c|c|c|}
\hline \multicolumn{3}{|c|}{ Baroco (déduction indirecte) } \\
\hline 1 & $\mathrm{MaN}$ & Prémisse \\
\hline 2 & MoP & Prémisse \\
\hline 3 & $\mathrm{NaP}$ & $\mathrm{C}(\mathrm{NoP})$ \\
\hline 4 & $\mathrm{MaN}$ & Réitération (1) \\
\hline 5 & $\mathrm{MaP}$ & PS1 $(3,4)$ \\
\hline 6 & MoP & Réitération (2) \\
\hline 7 & NoP & CTR \\
\hline
\end{tabular}

On voit que le modèle de Corcoran est assez exactement similaire à celui d'Ebbinghaus, si on présente le système déductif $D$ de cette manière, c'est-àdire sous la forme d'un ensemble de règles d'inférence définies à partir d'un langage donné. Pourtant cette présentation est, en toute rigueur, trompeuse (même si elle permet de rendre manifeste une forte similitude avec le calcul $\left.K_{s}\right)$. En effet, dans l'article de 1974, Corcoran, après avoir défini le langage $L$, ne pose pas à proprement parler des « règles d'inférence », ici indiquées par le symbole $\vdash$. Ces règles d'inférence sont plutôt basées sur la formulation première de « lois », appartenant à une sémantique nommée $S$, et d'emblée associée au système déductif $D$. Elles sont donc rendues au moyen du symbole $\vDash$, qui exprime la conséquence logique, et pas seulement la dérivabilité. La validité de ces lois est fondée par Corcoran sur la définition d'une fonction de vérité qui associe les phrases de $L$ à un « domaine » spécifique. Selon Corcoran, Aristote considère la valeur de vérité des propositions catégoriques non modales comme déterminée extensionnellement. Il se base alors sur ce fait pour relier les règles d'inférence à une fonction de vérité $V$ associée à une interprétation des éléments du langage $L$ qui fonde la possibilité pour les prémisses ou la conclusion d'un syllogisme d'être qualifiées de vraies. En fait, Corcoran se sert de la sémantique de la théorie des modèles de Tarski dans une forme abrégée. De façon générale, une interprétation $\boldsymbol{i}$ est, dans ce cadre, une fonction qui associe des signes à un domaine : le résultat de l'interprétation d'un terme $x$, c'est-à-dire $i x$, est une classe, à savoir un sous-ensemble spécifique du domaine de discours. Quand les signes sont des phrases, on définit une fonction de valuation $V^{t}$ (basée sur l'interprétation $i$ ) qui leur assigne alors des valeurs de vérité, qui dépendent de l'interprétation des termes. L'interprétation de « $A x y »$, c'est-à-dire $V^{t}(A x y)$, est vraie, si et seulement si la classe qui interprète $x$ est incluse dans la classe qui interprète $y$. La fonction d'interprétation relie des signes non interprétés à un domaine garantissant la possibilité d'une sémantique, conçue en termes de fonctions de vérité, à partir de laquelle Corcoran définit les notions de négation et de contradiction présentes dans les Premiers analytiques.

En d'autres mots, Corcoran suppose que le système de déduction naturelle qui se laisse dégager des Premiers analytiques possède une sémantique vérifonctionnelle sous-jacente. Cette sémantique est préfigurée par la fonc- 
tion d'interprétation. Plus généralement, Corcoran 1972 aussi bien que Smiley 1962 notent qu'Aristote utilise à la fois un système d'inférence et une méthode de contre-exemple (voir Smiley 1962 p. 62), et ils supposent que cette dernière requiert une sémantique vérifonctionelle. Or, si on suppose une telle sémantique formelle, on a besoin d'une preuve de complétude pour assurer que le système syllogistique inclut tout argument que la sémantique présente comme logiquement vrai. Rappelons que Corcoran 1972 réalise cette preuve. Néanmoins, cette manière d'envisager la question de la complétude est plutôt greffée au texte des Analytiques qu'elle n'y est préfigurée. L'approche opérative d'Ebbinghaus ne requiert aucune sémantique de ce type. Une manière d'esquisser la différence entre les deux approches consiste à dire que Corcoran et Smiley utilisent un système « hybride ». En effet, on remarquera, par exemple, que Corcoran 1972 p. 697 ajoute aux règles du système inférentiel une définition vérifonctionnelle de la négation d'une proposition. Il est intéressant d'y comparer les pures règles d'inférence qu'emploie Ebbinghaus pour définir la négation (règles $\left(D_{1}\right),\left(D_{2}\right),\left(D_{3}\right),\left(R_{4.1}\right),\left(R_{4.2}\right),\left(R_{4.3}\right)$ et $\left(R_{4.4}\right)$ ).

\section{Vers une autre compréhension de la question de la complétude}

Malgré tout, Ebbinghaus ne conteste pas qu'Aristote ait pu s'intéresser à la question de la complétude. Il écrit, au contraire, au moment où il rapporte la théorie du syllogisme au contexte pragmatique des jeux de discussions, qu'Aristote « s'intéresse beaucoup à la preuve de la complétude (Nachweis der Vollständigkeit) de son système, à cela que toutes les inférences passent par les figures répertoriées, que chaque preuve requiert trois termes...etc. ${ }^{29}$. Puisque cependant, il ne s'occupe pas, dans cet article, de la question de la vérité ${ }^{30}$, sinon incidemment et pour la rejeter hors de son propos, étant donné que « le concept de vérité est éliminé de la syllogistique ${ }^{31}$, alors on doit en conclure que l'intérêt d'Aristote pour une preuve de complétude doit être pensé autrement qu'à partir de la question de la vérité, donc autrement que Corcoran ne la pose, en termes de « fonction de vérité ». Ebbinghaus perçoit la raison de cet intérêt dans le fait qu'Aristote a inventé, avec la théorie du syllogisme, « un procédé au moyen duquel il devient possible de décider de la validité ou de la non-validité de chaque étape de la discussion $\gg^{32}$. La

29. Ebbinghaus 2016 p. 56

30. «Les propriétés « vrai » et « faux » ne sont pas définies pour les figures du calcul $[K]$ et ne peuvent être définies de façon opérative », p. 38.

31. Voir p. 60.

32. Voir p.49. Le verbe «entscheiden 》 (décider) et l'expression «Entscheidungsverfahren » (procédé de décision) employés dans la même page par Ebbinghaus font écho au problème de la décision ( «Entscheidungsproblem ») posé par Hilbert, problème qui trouverait, en quelque sorte, dans la syllogistique d'Aristote une solution positive. Notons qu'il s'agit là d'une théorie logique ne permettant pas de formaliser les relations, contrairement à 
« question de la complétude » est en quelque sorte déplacée et n'est plus à comprendre selon le sens qu'elle revêt lorsqu'on commence par séparer les règles d'inférence des questions sémantiques. Un système d'inférence ne sera pas qualifié de « complet » s'il est corrélé à une structure ensembliste dont il restituerait entièrement les relations inclusives. Il le sera, dans un autre sens, s'il est associé à la preuve que toutes les inférences qu'il rend possibles sont susceptibles d'être normalisées, c'est-à-dire ramenées à des figures canoniques qui correspondent, non pas à des règles évidentes conservant la vérité, mais à des assomptions fondamentales instituant le cadre dans lequel une discussion entre individus raisonnables devient possible. Tel est le sens du concept d'admissibilités ${ }^{33}$ qui rend possible de penser la notion de validité comme, en quelque sorte, immanente aux inférences elles-mêmes. Ebbinghaus a l'intuition géniale qu'en réduisant l'ensemble des syllogismes à quelques figures canoniques, moyennant l'usage des règles de conversion, Aristote n'a pas besoin d'une preuve de complétude. Dans les Analytiques, l'instrument inférentiel constitue l'objet même de l'analyse logique, indépendamment de

la logique du premier ordre, dont Church a établi l'indécidabilité, et que ce n'est évidemment pas le type de théorie logique que Hilbert avait en vue lorsqu' il formulait le « problème de la décision ». D'ailleurs, Ebbinghaus parle de la décidabilité d'une inférence et non pas de celle d'une proposition : il ne s'agit pas, pour Aristote, de se demander si on peut décider de la dérivabilité ou de la non-dérivabilité de toute proposition, mais plutôt si on peut décider de la validité de toute inférence. Or, c'est justement possible si toute inférence valide constitue un détour réductible au moyen d'une preuve d'admissibilité. Le « flottement » entre le problème de la décision et celui de la complétude tient sans doute au fait que l'approche d'Ebbinghaus présuppose une manière de penser la signification qui differe de celle en fonction de laquelle ces problèmes ont été formulés par Hilbert. On peut dire que ce que fait Aristote concerne la complétude si on comprend celle-ci comme détermination complète de l'ensemble des inférences du point de vue de la validité. Cependant, il est sans doute plus approprié de parler de l'admissibilité de toutes les inférences valides relativement aux règles fondamentales de la théorie du syllogisme.

33. Il est important de savoir que le concept d'admissibilité a eu une importance décisive dans la construction d'une sémantique de la théorie de la preuve, notamment à travers le « principe d'inversion » qu'il permet de fonder, que Lorenzen formule en 1953 et que Dag Prawitz reprendra en 1965 pour développer l'idée que la signification d'un connecteur logique est donnée à travers des règles, à savoir les règles dites d'introduction et d'élimination de ce connecteur (idée que Gentzen formulait dès 1934). Si le travail d'Ebbinghaus est en quelque sorte traversé par des idées novatrices, et en chantier, sur la nature de la signification en général, mais plus particulièrement celle des constantes logiques, c'est d'une manière qui n'est pas toujours totalement maîtrisée, ce dont témoigne, par exemple, la note de bas de page concernant la dialogique, dont le contenu est suggéré, de l'aveu d'Ebbinghaus, par Lorenzen, mais qui s'intègre difficilement au contexte de l'article. Il nous semble qu'un des intérêts de ce dernier ressort justement de l'impression d'indécision et de flottement qui s'en dégage et qui est l'indice d'une bifurcation hésitante vers une approche nouvelle de la notion de validité, encouragée par la lecture toute «matinale » qu'elle rend possible du texte des Analytiques. Sur le développement de la sémantique de la théorie de la preuve à partir des travaux de Lorenzen, voir Schroeder-Heister 2018 [2012]. 
toute perspective référentielle, renvoyée en dehors du domaine propre de la logique. Si Corcoran a bien perçu que les syllogismes étaient des règles d'inférence, il s'en est tenu à l'idée que ces règles devaient servir, en dernière instance, à transporter et à conserver la vérité, qui seule fonde, à ses yeux, leur validité. Ebbinghaus va, pour sa part, beaucoup plus loin, en suggérant qu'Aristote pense la validité syllogistique à travers l'analyse des inférences elles-mêmes et de leur réductibilité aux règles d'inférence canoniques que sont les syllogismes parfaits et les règles de conversion. C'est donc, en somme, le concept d'admissibilité qui permet de nommer la manière spécifique dont Aristote élabore la preuve de la complétude de son système, la mise en œuvre de cette preuve correspondant à celle de réduction des détours. On pourrait certes appeler ici «preuve de complétude » une preuve que la validité de tous les « coups » possibles dans le cadre d'un jeu peut faire l'objet d'une « décision » fondée sur la construction d'une relation d'équivalence entre les règles d'inférence auxquelles correspondent ces « coups » et les règles canoniques qui constituent fondamentalement le cadre du jeu, mais il serait plus naturel de parler de « preuve d'admissibilité » en ayant bien en tête qu'établir l'admissibilité de schémas inférentiels suffit pour fournir ce que Corcoran prétendait réaliser à travers sa preuve de complétude et que c'est exactement ce que fait Aristote dans les Premiers analytiques. Prouver l'admissibilité d'un syllogisme est, au demeurant, un procédé plus général que celui qui consiste à admettre la validité d'un syllogisme imparfait en raison de sa perfectibilité. Gisela Striker 1999 a indiqué la nécessité de penser une telle notion générale, en s'appuyant sur le fait qu'après tout, les Premiers analytiques contiennent d'autres procédés que la réduction à un syllogisme parfait pour fonder la validité des syllogismes, à savoir l'ecthèse, la reductio ad impossibile, mais aussi la réduction des syllogismes de la première et de la deuxième figures à ceux de la troisième (I, 45, 50b-51b).

On notera, enfin, que la notion de réductibilité à la première figure peut être généralisée pour n'importe quel syllogisme. Cette généralisation provient de la compréhension que prouver la réductibilité d'un syllogisme quelconque revient à prouver que ce syllogisme est admissible, s'il est éliminable à travers l'utilisation de quelques règles basiques ou canoniques. L'admissibilité permet donc de discerner, parmi tous les arguments possibles, ceux qui sont logiquement valides, c'est-à-dire les syllogismes. Cela suffit à Ebbinghaus pour atteindre sa visée métalogique (analogue à la preuve de complétude de Corcoran 1972). Il aurait certainement pu élaborer en détail un procédé pour reconstruire la méthode du contre-exemple, à l'intérieur du cadre qu'il était en train de développer, mais il ne l'a pas fait - et a tristement disparu du monde académique après la publication du texte que nous sommes en train de discuter. Quoi qu'il en soit, la procédure consistant à produire des contreexemples ne doit pas nécessairement être comprise en termes de fonction 
de vérité : Marion \& Rückert 2016 ont montré que cette méthode pouvait être comprise comme faisant partie de la signification inférentielle des quantificateurs chez Aristote. Ce qu'on peut sans doute objecter à l'approche d'Ebbinghaus, c'est que, contrairement à celui de Corcoran, son système ne paraît pas exclure les prémisses de la forme « Homme est prédiqué de tout homme » - et du moins, que c'est une question sur laquelle il ne se prononce pas. Ce point a été signalé par Smiley 1973, note de fin 5, qui, à cette occasion, reconnaît le travail d'Ebbinghaus, et qui est, à notre connaissance, le seul, avec Patzig 1969 et, plus récemment, Striker 1999, 2009, à l'avoir fait.

S'il vaut la peine de lire (ou de relire) aujourd'hui cette monographie de 1964, ce n'est toutefois peut-être pas tant pour lui restituer la part d'influence qui fut la sienne, d'une manière plus ou moins souterraine, dans le domaine des études des écrits logiques d'Aristote, que pour redécouvrir, dans son éclat naissant, une intuition lumineuse relative à l'essence de la logique, intuition qui pourrait, dans sa discrétion même, ouvrir la perspective de nouveaux développements audacieux, s'inscrivant dans la continuité des textes de l'Organon. 


\section{Annexe : tableau comparatif des reconstructions proposées pour les preuves de Festino et de Baroco ${ }^{34}$.}

Festino (preuve directe)

\begin{tabular}{|c|c|c|c|c|c|}
\hline \multicolumn{3}{|c|}{ EBBINGHAUS } & \multicolumn{3}{|c|}{ CORCORAN } \\
\hline$l$ & $\mathrm{MeN}$ & & 1 & $\mathrm{MeN}$ & prémisse \\
\hline$m$ & $\mathrm{MiP}$ & & 2 & $\mathrm{MiP}$ & prémisse \\
\hline$l \rightarrow n$ & $\mathrm{NeM}$ & $\mathrm{R}_{1}$ & 3 & $\mathrm{NeM}$ & C1 (1) \\
\hline & & & 4 & MiP & $\begin{array}{l}\text { Réitération } \\
\text { (2) }\end{array}$ \\
\hline$n, m \rightarrow n+1$ & $\mathrm{NoP}$ & $\mathrm{R}_{6}$ & 5 & $\mathrm{NoP}$ & PS4 $(3,4)$ \\
\hline
\end{tabular}

\section{Baroco (preuve indirecte)}

\begin{tabular}{|c|c|c|c|c|c|}
\hline \multicolumn{3}{|c|}{ EBBINGHAUS } & \multicolumn{3}{|c|}{ CORCORAN } \\
\hline$l$ & $\mathrm{MaN}$ & & 1 & $\mathrm{MaN}$ & prémisse \\
\hline$m$ & MoP & & 2 & MoP & prémisse \\
\hline$n$ & $\mathrm{NaP}$ & $\begin{array}{l}\text { prémisse } \\
\text { ajoutée }\end{array}$ & 3 & $\mathrm{NaP}$ & $\mathrm{C}(\mathrm{NoP})$ \\
\hline & & & 4 & $\mathrm{MaN}$ & $\begin{array}{l}\text { Réitération } \\
\text { (1) }\end{array}$ \\
\hline$l, n \rightarrow n+1$ & $\mathrm{MaP}$ & $\mathrm{R}_{2}$ & 5 & $\mathrm{MaP}$ & PS1 $(3,4)$ \\
\hline$n+1, m \rightarrow n+2$ & $\Lambda$ & $\mathrm{D}_{1}$ & 6 & MoP & $\begin{array}{l}\text { Réitération } \\
\text { (2) }\end{array}$ \\
\hline $\begin{array}{l}(n \rightarrow n+2) \rightarrow \\
n+3\end{array}$ & $\mathrm{NoP}$ & $\mathrm{R}_{4.1}$ & 7 & NoP & CTR \\
\hline
\end{tabular}

34. Ces deux exemples font partie de ceux qui ont été choisis par Ebbinghaus 1964, et sont différents de ceux choisis par Corcoran 1974. Nous avons donc indiqué, en vue de la comparaison, les règles qui auraient servi à reconstruire les exemples en question dans le système de Corcoran en adoptant une notation différente de la sienne (cf. note 27). 


\section{BIBLIOGRAPHIE}

Corcoran, J. 1972 : « Completeness of an Ancient Logic », Journal of Symbolic Logic, 37 (1972), p. 696-702.

http://www.jstor.org/stable/2272415 (DOI : 10.2307/2272415)

— 1973 : « A Mathematical Model of Aristotle’s Syllogistic », Archiv für Geschichte der Philosophie, 55 (1973), p. 191-219 (DOI : 10.1515/agph.1973.55.2.191).

— 1974a : « Aristotle’s Natural Deduction System », dans J. Corcoran (éd.), Ancient Logic and its Modern Interpretations, Dordrecht, 1974, p. 85-131 (Synthese Historical Library, 9).

- 1974b : « Aristotelian Syllogisms: Valid Arguments or True Universalized Conditionals? », Mind, New Series, 83 (1974), p. 278-281. http://www.jstor.org/stable/2252914

— 1994: « The Founding of Logic. Modern Interpretations of Aristotle's Logic », Ancient Philosophy, 14/Special Issue (1994), p. 9-24.

Crubellier, M. 2011 : «Du sullogismos au syllogisme », Revue philosophique de la France et de l'étranger, 136/1 (2011), p. 17-36. https://www.cairn.info/revuephilosophique-2011-1-page-17.htm (DOI : 10.3917/rphi.111.0017)

- 2014 (trad.) : Aristote, Premiers Analytiques, traduction, introduction, notes, commentaire et bibliographie, Paris, 2014 (GF).

Ebbinghaus, K. 1964 : Ein formales Modell der Syllogistik des Aristoteles, Göttingen, 1964 (Hypomnemata, Untersuschungen zur Antike und zu ihrem Nachleben, 9).

- 2016: Un modèle formel de la syllogistique d'Aristote, traduit par C. Lion, London, 2016 (Cahiers de Logique et d'Épistémologie, 24).

FILlion, N. 2007 : « Two accounts of Aristotle's Logic », Seminar on Aristotelian Logic, Ph.D in Philosophy, University of Western Ontario, Faculty of Arts, 16 avril 2007, en ligne https://www.yumpu.com/en/document/view/36257366/ two-accounts-of-aristotles-logic-nicolas-fillion.

Gourinat, J.-B. 2011 : « Aristote et la "logique formelle moderne": sur quelques paradoxes de l'interprétation de Eukasiewicz », Philosophia Scientiae, 15/2 (2011), p. 69-101.

http://journals.openedition.org/philosophiascientiae/654 (DOI : 10.4000/ philosophiascientiae.654)

KAPP, E. 1942 : Greek foundation of traditional logic, New-York, 1942.

Lorenzen, P. 1969 : Einführung in die operative Logik und Mathematik, Berlin - Göttingen - Heidelberg, 1969 (Die Grundlehren der mathematischen Wissenschaften in Einzeldarstellungen mit besonderer Berücksichtigung der Anwendungsgebiete, 78).

ŁUKASIEWICZ, J. 1972 : La syllogistique d'Aristote dans la perspective de la logique formelle moderne, trad. de F. Zalawsky, Paris, 1972 ( $2^{\mathrm{e}}$ éd. 2010).

Marion, M. \& H. Ruckert, 2016 : « Aristotle on universal quantification: a study from the point of view of game semantics » History and Philosophy of Logic, 37/3 (2016), p. 201-209.

PatzIG, G. 1959 : Die aristotelische Syllogistik, logisch-philologische Untersuchungen über das Buch A der "Ersten Analytiken", Göttingen, 1959 (Abhandlungen der 
Akademie der Wissenschaften in Göttingen : Philologisch-Historische Klasse. 3. Folge, 42).

- 1968: Aristotle's theory of the syllogism: a logico-philosophical study of Book A of the Prior Analytics, trans. by J. Barnes, Dordrecht, 1968 (Synthese Library).

Prawitz, D. 1965 : Natural deduction : a proof-theoretical study, Stockholm, 1965 (Acta Universitatis Stockholmiensis).

Rahman, S., Z. McConaughey \& M. Crubellier, en préparation : « Dialectic, the Dictum de Omni and Ecthesis »(<halshs-01652027> https://halshs. archives-ouvertes.fr/halshs-01652027/document).

Rahman, S. \& G. Nzokou, 2015 : « Les racines dialectiques de la syllogistique aristotélicienne. Identité et quantification universelle », Conférence Ada-Lacto, MESHS Lille. », 2015 (<halshs-01315118> https://halshs.archives-ouvertes. $\mathrm{fr} /$ halshs-01315118).

Schroeder-Heister, P. 2018 : « Proof-Theoretic Semantics », The Stanford Encyclopedia of Philosophy, Edward N. Zalta (éd.), 2018 [2012].

https://plato.stanford.edu/archives/spr2018/entries/proof-theoretic-semantics/.

Smiley T. 1962 : «Syllogism and quantification », The Journal of Symbolic Logic, 27/1 (1962), p. 58-72.

- 1973 : «What is a Syllogism », Journal of Philosophical Logic, 2/1 (1973), p. 136-154.

Smith, R. 1982 : «What is Aristotelian Ecthesis? », History and Philosophy of Logic, 3 (1982), p. 113-127. https://doi.org/10.1080/01445348208837035

- 1983 : « Completeness of an Ecthetic Logic », Notre Dame Journal of Formal Logic, 24 (1983), p. 224-232.

- 1989 (trad.) : Aristotle's Prior Analytics, transl. with an introd., notes \& comm., Indianapolis, 1989.

Striker, G. 1999 : « Perfection and Reduction in Aristotle's Prior Analytics », dans M. Frede \& G. Striker (éd.), Rationality in Greek Thought, Oxford, 1999, p. 203-219.

- 2009 (trad.) : Aristotle, Prior Analytics : Book I (translation and commentary), Oxford, 2009.

Tном, P. 1981 : The Syllogism, Munich, 1981 (Analytica. Investigations in Logic, Ontology and the Philosophy of Langage). 